\title{
Performance Analysis of Pseudorange Error in STAP Beamforming Algorithm for Array Antenna
}

\author{
Kihoon Lee ${ }^{\dagger}$, Hyungmin So, Kiwon Song \\ Navigation Department, Agency for Defense Development, Daejeon 305-600, Korea
}

\begin{abstract}
The most effective method to overcome GPS jamming problem is to use an adaptive array antenna which has the capability of beamforming or nulling to a certain direction. In this paper, Space Time Adaptive Processing (STAP) beamforming algorithm of four elements array antenna will be designed and the anti-jamming performance will be analyzed. According to the analysis, the signal to noise ratio (SNR) and anti-jamming performance can be enhanced by beamforming algorithm. Also, the time tap effect of STAP algorithm will be analyzed theoretically and verified with array antenna modeling and simulation. Specially, the cautious selection of reference time tap in STAP can prevent the degradation of position accuracy performance.
\end{abstract}

Keywords: STAP, beamforming, code delay distortion, carrier phase distortion

\section{INTRODUCTION}

GPS receivers use very weak satellite signals of $1 \mathrm{pW}$. Accordingly, they cannot track satellite signals even at weak interference signals, and this has been a major problem (Parkinson et al. 1996, Kaplan \& Hegarty 2006, Rhee \& Seo 2013). For example, 1 Watt portable jammer can interrupt the normal operation of GPS receivers within a $50 \mathrm{~km}$ radius. The most effective method for overcoming this problem is to spatially eliminate interference signals using array antennas (Johnson \& Dudgeon 1993, Fante \& Vaccaro 2000, Haefner et al. 2003, Kaplan \& Hegarty 2006).

An array antenna is a system that can control gains depending on incident directions by generating final output through multiplying a number of input signals by a weight vector. If this is applied to GPS anti-jamming devices, the gain in the satellite direction can be conserved while minimizing the gain in the incident direction of a jammer. The performance of an array antenna is basically determined by the number of antenna elements and the arrangement

Received Jan 22, 2014 Revised Feb 06, 2014 Accepted Feb 12, 2014 †Corresponding Author

E-mail: kihoon@add.re.kr

Tel: +82-42-821-4468 Fax: +82-42-823-3400 type. In theory, it is known that when the number of antenna elements is $\mathrm{N}$, the number of jammers, to which anti-jamming can be applied, is $\mathrm{N}-1$. Also, there are the arrangement types such as Uniform Linear Array, Uniform Rectangular Array, Uniform Circular Array, and Single Ring Cylindrical Array. In this study, a four-element circular array antenna system, which can process a maximum of three broadband jamming signals, is considered in order to minimize the size, as shown in Fig. 1. In addition, the distance between antenna elements is a major design factor, and the half wavelength distance principle, which is commonly applied, was used considering mutual coupling and nulling regions (Johnson \& Dudgeon 1993).

In the previous papers, an Space Time Adaptive Processing (STAP) algorithm for array antenna systems, which was

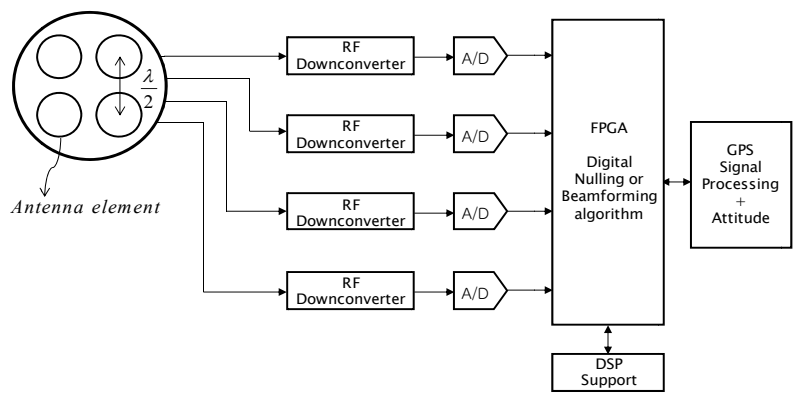

Fig. 1. Digital anti-jamming array antenna system. 
extended to time and space domains to extend narrowband anti-jamming number, has been used. In this regard, a pseudorange tracking error, which determines the positioning accuracy of a GPS receiver, occurs. But the cause of this error has not been analyzed (Ward 1994, Moore 2002, Carlson et al. 2003, Fante et al. 2004). In this study, the cause of pseudorange tracking error during the use of an STAP algorithm was analyzed, and the performance analysis of the STAP algorithm which a beamforming algorithm has been added was carried out in terms of signal to noise ratio and changes in the characteristics of satellite signals.

The contents of this paper are as follows. In Chapter 2, an STAP beamforming algorithm was derived, and in Chapter 3 a tap selection technique for preventing pseudorange distortion was proposed. In Chapter 4 , the results of the simulation were presented, and conclusions were included in Chapter 5.

\section{STAP BEAMFORMING ALGORITHM}

\subsection{Optimal Weight Vector Algorithm For Array Antenna}

An array antenna system generates final output by multiplying a number of input signals by a weight vector and adding them together. In this regard, the algorithm for weight vector determination is a constrained optimization problem for the minimization of the objective function, $f(\mathrm{w})$, depending on the constraint condition of the variable, $\mathrm{w}$, as shown in Eq. (1) (Johnson \& Dudgeon 1993).

$$
\min _{\mathrm{w}} f(\mathrm{w}) \text { subject to } g(\mathrm{w})=0
$$

The solution of this constrained optimization problem is one of the stationary points of the Lagrangian, and can be obtained using a Lagrangian multiplier (Applebaum \& Chapman 1976). First, the Lagrangian of Eq. (1) can be expressed as Eq. (2), and the Lagrangian multiplier, $\lambda_{0}$, of the stationary points of this can be expressed as Eq. (3).

$$
\begin{aligned}
& L(\mathrm{w}, \lambda)=f(\mathrm{w})+\lambda g(\mathrm{w}) \\
& \nabla_{\mathrm{w}} L\left(\mathrm{w}_{0}, \lambda_{0}\right)=0
\end{aligned}
$$

The input value measured at the I/Q channel of the fourelement array antenna is defined as $\mathrm{x}=\left[\begin{array}{llll}\mathrm{x}_{1} & \mathrm{x}_{2} & \mathrm{x}_{3} & \mathrm{x}_{4}\end{array}\right]^{T}$, and the constraint condition of the weight vector, $\mathrm{w}$, is defined as shown in Eq. (4).

$$
C \mathrm{w}=c
$$

where $C$ represents the constraint matrix, and c represents the constraining column vector. As the output of the array antenna is $f(\mathrm{w})=\mathrm{w}^{H} \mathrm{x}$, the expected value of the output power can be expressed as Eq. (5).

$$
\text { Output Power }=E\left[\left|\mathrm{w}^{H} \mathrm{x}\right|^{2}\right]=\mathrm{w}^{H} R \mathrm{w}
$$

where $E[\cdot]$ represents the expected value, $R=E\left[\mathrm{xx}^{H}\right]$, and $\mathrm{x}^{H}$ represents the conjugate transpose. Using the variables defined above, the constrained optimization problem for the minimization of the output power can be expressed as Eq. (6).

$$
\min _{\mathrm{w}} \mathrm{w}^{H} R \mathrm{w} \text { subject to } \mathrm{Cw}=c
$$

To obtain the solution of Eq. (6), a Lagrangian multiplier is calculated. Then the weight vector, $\mathrm{w}_{\mathrm{o}^{\prime}}$, and the power, $P$, which are the optimal solution, can be calculated as shown in Eqs. (7) and (8).

$$
\begin{gathered}
\mathrm{w}_{\mathrm{o}}=R^{-1} C^{H}\left(C R^{-1} C^{H}\right)^{-1} c \\
P=c^{H}\left(C R^{-1} C^{H}\right)^{-1} c
\end{gathered}
$$

The anti-jamming algorithm of the array antenna basically follows the above equations for deriving optimal solution, and the implementation methods are broadly divided into two categories. In the first method, the correlation matrix, $R$, of an input value is estimated, and the inverse matrix is obtained. Then the weight vector is directly calculated as shown in Eq. (7). In the second method, the weight vector is estimated so that output can be minimized, using a Least Mean Square algorithm without the estimation of the correlation matrix, $R$ (Frost 1972, Lorenzo et al. 2005).

\subsection{STAP Beamforming Algorithm}

The STAP input shown in Eq. (9) is used for STAP algorithm that is extended to time and space domains, which is extended to the time domain by the number of taps $\mathrm{T}$ when the input, $\mathrm{x}=\left[\begin{array}{llll}\mathrm{x}_{1} & \mathrm{x}_{2} & \mathrm{x}_{3} & \mathrm{x}_{4}\end{array}\right]^{T}$, of the four-element array antenna is used. The remaining processes for calculating the optimal weight vector are identical to the method explained above. For an array antenna with more than four elements, extension can be performed in a similar way.

$$
\mathrm{x}=\left[\begin{array}{lllllllll}
\mathrm{x}_{11} & \mathrm{x}_{12} \ldots \mathrm{x}_{1 \mathrm{~T}} & \mathrm{x}_{21} & \mathrm{x}_{22} \ldots \mathrm{x}_{2 \mathrm{~T}} & \ldots & \mathrm{x}_{41} & \mathrm{x}_{42} \ldots \mathrm{x}_{4 \mathrm{~T}}
\end{array}\right]^{T}
$$

The degree of freedom of this STAP algorithm is basically obtained by subtracting the number of constraint 
conditions from the number of free weight vector elements. It is expressed as the following equation, where $\mathrm{N}-1$, which is the degree of freedom of the spatial nulling algorithm that performs spatial filtering, is extended by $T$ (number of taps) times.

$$
D O F_{S T A P}=N T-1
$$

For the above STAP algorithm, the degree of freedom is extended by $\mathrm{T}$ times. Thus, it is robust to a narrowband jammer, which consumes one degree of freedom, and can counteract $N T$-1narrowband jammers in theory (Gupta \& Ksienski 1983, Berefelt et al. 2003, Vo et al. 2007).

The STAP beamforming algorithm uses a Maximum Signal to Interference Ratio method, which calculates the weight vector considering the steering vector, $\mathrm{s}$, in order to conserve the gain and phase in a specific direction (satellite direction) or a specific frequency in Eq. (4) for constraint condition. In this regard, the constraint matrix, $C$, is identical to the steering vector, $s$, and the final optimal weight vector can be expressed as Eq. (11).

$$
\mathrm{w}_{\mathrm{o}}=\frac{R^{-1} s}{s^{H} R^{-1} s}
$$

The STAP beamforming algorithm increases the gain in a specific direction, and the theoretical gain can be expressed as Eq. (12). In addition, to increase the effect at a specific frequency, thermal noise or jamming signals can be additionally eliminated by applying constrained conditions to a specific tap.

$$
d B=10 \times \log (N)
$$

For the above STAP beamforming algorithm, the gain in a specific direction can be achieved by controlling the parts of the steering vector, $\mathrm{s}$, which are related with the array antenna. In addition, it needs to be designed considering the effect of a tap, which is a time delay, and this is analyzed in Chapter 3. Using the STAP beamforming algorithm considering this, a simulation is performed, and the results are presented in Chapter 4.

\section{TAP SELECTION IN STAP BEAMFORMING}

In this chapter, the distortion characteristics of a pseudorange, which affects the positioning error of a GPS receiver when STAP with an additional filter is applied in the time domain, were analyzed. The tap of STAP is a time

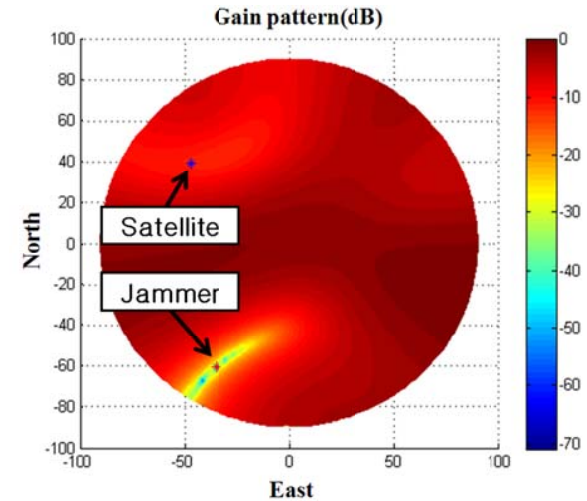

Fig. 2. Gain pattern of STAP when SV and jammer exist.

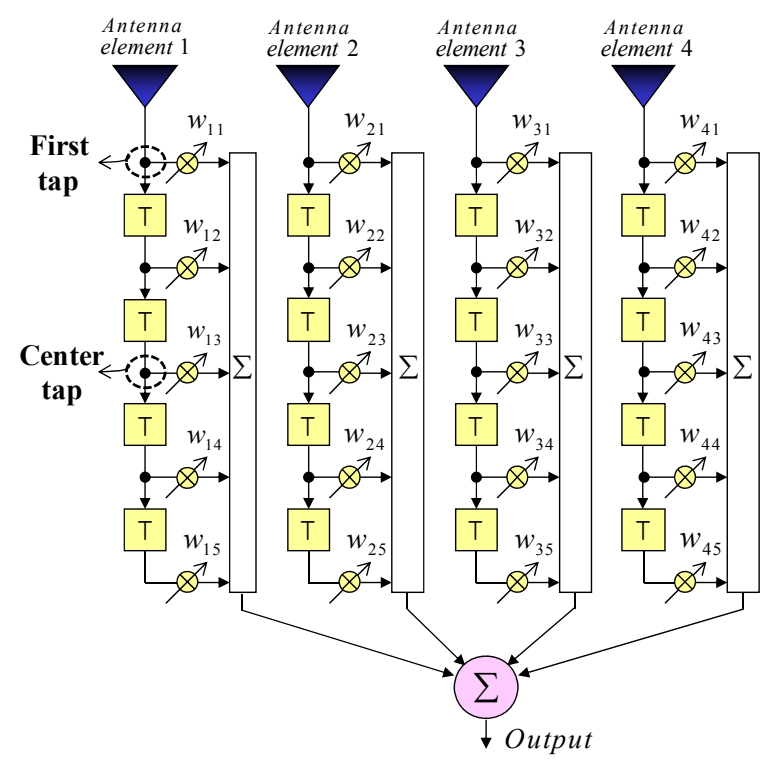

Fig. 3. Space Time Adaptive Processing structure with 5 taps and 4 antennas.

delay, and a GPS receiver is very sensitive to a time delay. Especially, for the application of STAP, the effects could be significant depending on the selection of a reference tap. For example, when a satellite and a jammer are arranged as shown in Fig. 2, and the first tap in Fig. 3 is selected as the reference tap, the front and rear shapes of the Cross Correlation Function (CCF), which is the correlation function of satellite signals, are deformed and distorted as shown in Fig. 4. In this regard, when the general Early minus Late pseudorange tracking technique was used, a pseudorange error of about $30 \mathrm{~m}$ was obtained as shown in Fig. 4. This error component is problematic because each satellite has a different pseudorange error.

The CCF distortion of satellite signals is due to the nonlinear phase effect of a transversal filter that has time 


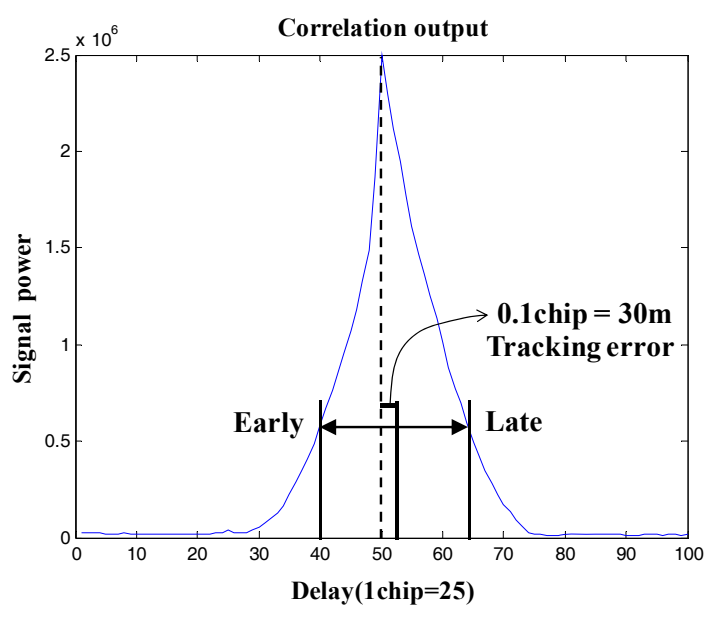

Fig. 4. Distorted CCF of SV signal.

delay taps. The linear phase of the filter represents the case when the group delay, G, of the filter is a constant as shown in Eq. (13).

$$
G=\frac{\Delta \phi}{\Delta f}=\text { Constant }
$$

where $\Delta f$ represents the frequency variation, and $\Delta \phi$ represents the phase variation.

In the case of a nonlinear phase, each input frequency has a different time delay because a phase variation for a frequency variation is not linearly maintained. Therefore, within a satellite signal bandwidth, each time delay is different depending on the frequency, and the distortion of $\mathrm{CCF}$ occurs when correlation functions using replica signals are calculated. To verify this, CCF was obtained by inputting only satellite signals and passing them through a 41st-order transversal filter. When the signals were passed through a nonlinear phase filter as shown in Fig. 5, the front and rear shapes of CCF were non-symmetric, which resulted in a pseudorange error. In contrast, when the signals were passed through a linear phase filter as shown in Fig. 6, a time delay occurred, but the front and rear shapes of CCF were symmetric with the same power, which did not result in a pseudorange error when the Early minus Late tracking technique was used.

From the above discussion, it was found that for a transversal filter with time taps such as the case of an STAP algorithm, the filter should have linear phase characteristics. For an FIR filter with time delay taps to have linear phase characteristics, the filter coefficient should consist of symmetry (Haykin 1996, Bellanger 2000). This
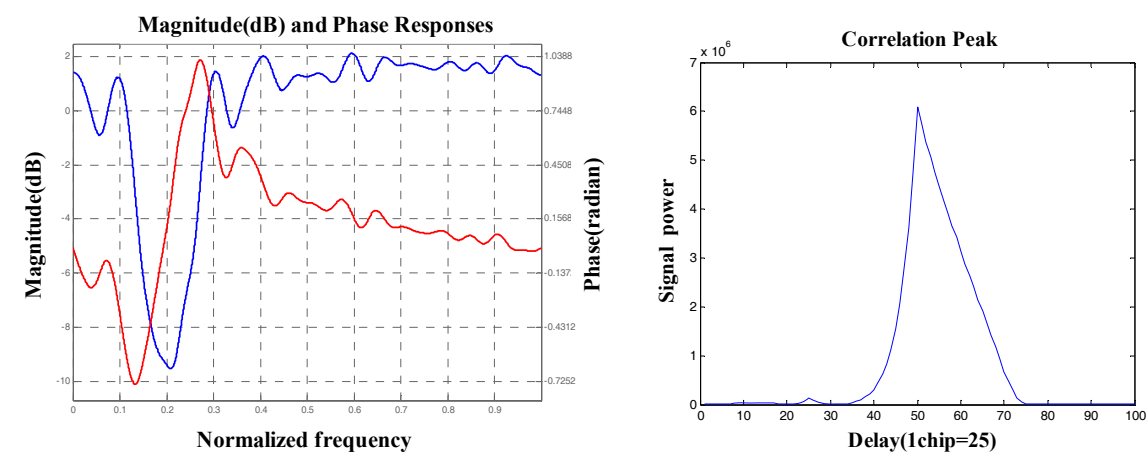

Fig. 5. Nonlinear phase 41 taps FIR filter bode plot (blue: amplitude, red: phase) and non-symmetric CCF.
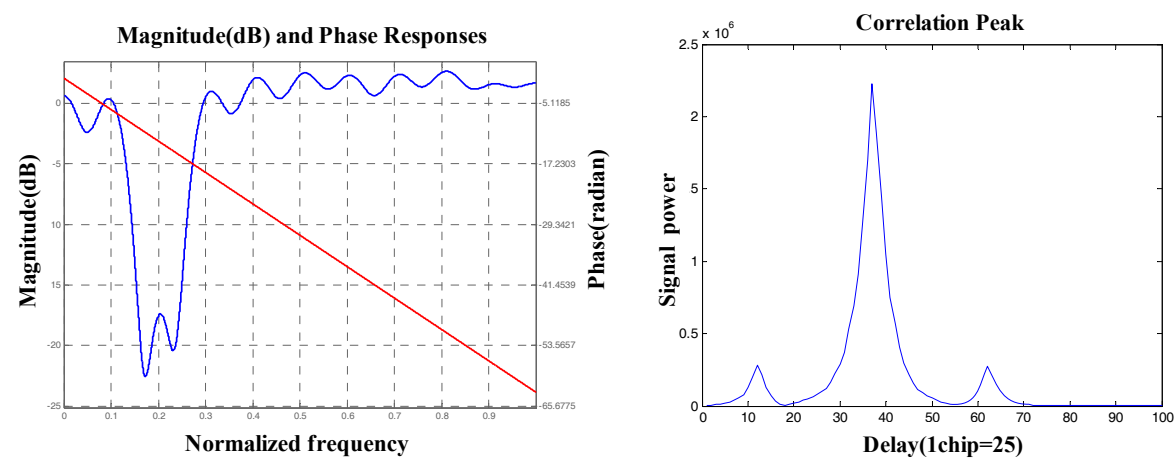

Fig. 6. Linear phase 41 taps FIR filter bode plot (blue: amplitude, red: phase) and symmetric CCF. 
also applies to the STAP algorithm, and it indicates that linear phase characteristics can be obtained when the center tap is selected in the process of obtaining a weight vector using a correlation matrix, as shown in the following equations. In other words, the weight vector, $\mathrm{w}_{\mathrm{o}}$, is to select a specific column of $R^{-1}$, and only the center column of $R^{-1}$ has symmetry characteristics.

$R:$ Symmetric matrix (Hermitian)

$R^{-1}$ : Symmetric matrix (Hermitian)

$\mathrm{w}_{\mathrm{o}}=\frac{R^{-1} s}{s^{H} R^{-1} S}$ : Symmetric if $s=\left[0 \cdots s_{1} \cdots 00 \cdots s_{2} \cdots 00 \cdots s_{3} \cdots 00 \cdots s_{4} \cdots 0\right]^{T}$

where $s$ represents the vector that located the satellite direction steering elements, $s_{1}, s_{2}, s_{3}, s_{4}$, at the center tap.

Fig. 7 shows the CCF correlation function of the satellite obtained after eliminating the jamming signals when the weight vector that selected the center tap was used so that the STAP algorithm could have linear phase characteristics. As shown in the figure, the Early and Late correlation values were symmetric, and thus pseudorange distortion did not occur. On the other hand, there was some time delay, but this is not problematic because it is common to every satellite. In conclusion, the number of taps for the STAP algorithm should be an odd number, and satellite navigation errors can be minimized when the center tap is selected as the reference tap.

\section{SIMULATION}

In this chapter, a simulation was performed by locating jamming signals at an azimuth angle of $0^{\circ}$ and an elevation

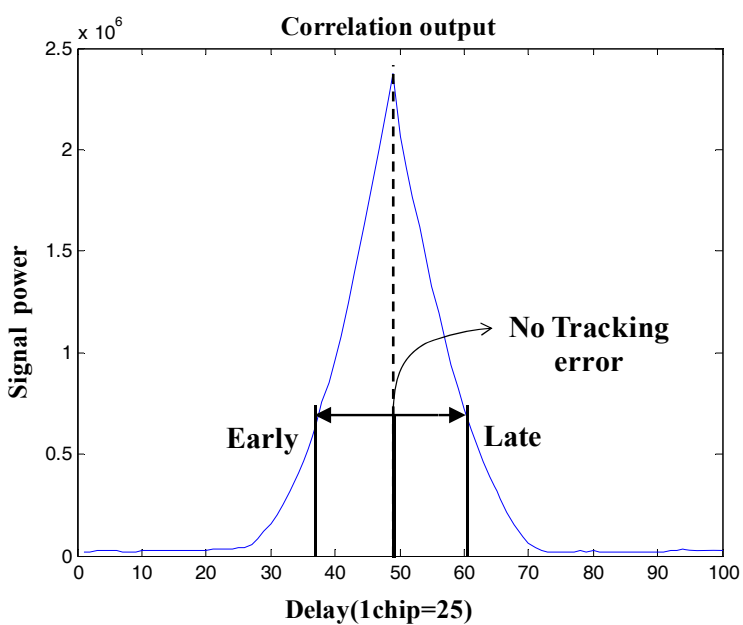

Fig. 7. CCF of Linear phase STAP filter. angle of $10^{\circ}$, and by locating satellite signals at an azimuth angle of $180^{\circ}$ and an elevation angle of $50^{\circ}$ to examine STAP beamforming effects under the condition summarized in Table 1.

Figs. 8 and 9 show the gain pattern and phase pattern when a simple power minimization technique without beamforming was applied. Jamming signals were completely eliminated, but a phase shift of $-60^{\circ}$ occurred at an azimuth angle of $180^{\circ}$ and an elevation angle of $50^{\circ}$, which was the satellite direction. On the other hand, the satellite signal SNR was calculated to be about $16 \mathrm{~dB}$ as shown in Fig. 10. This was determined by the gain pattern shown in Fig. 8, and was similar to the input signal SNR ( $15 \mathrm{~dB}$ ) of a single antenna in Table 1. In other words, the satellite signal SNR was normal, but the phase value changed depending on the incident direction of satellite signals as shown in the phase pattern. Thus, it would be problematic for systems using a carrier phase.

Figs. 11 and 12 show the gain pattern and phase pattern for the STAP technique using beamforming. For the gain pattern in Fig. 11, the gain in the satellite direction was about $6 \mathrm{~dB}$. From this figure, it was found that the maximum gain was maintained in the satellite direction. The phase pattern was calculated as shown in Fig. 12, and it was

Table 1. Simulation conditions.

\begin{tabular}{lc}
\hline \multicolumn{1}{c}{ Conditions } & Description \\
\hline Satellite signal SNR (Single antenna) & $15 \mathrm{~dB}$ \\
Jammer JNR & $20 \mathrm{~dB}$ \\
Antenna element number & 4 \\
STAP's tap number & 5 \\
Array type & Circular \\
Array distance & $9.5 \mathrm{~cm}$ \\
IF frequency & $0 \mathrm{~Hz}($ Baseband) \\
Sampling frequency & $20 \mathrm{MHz}$ \\
\hline
\end{tabular}

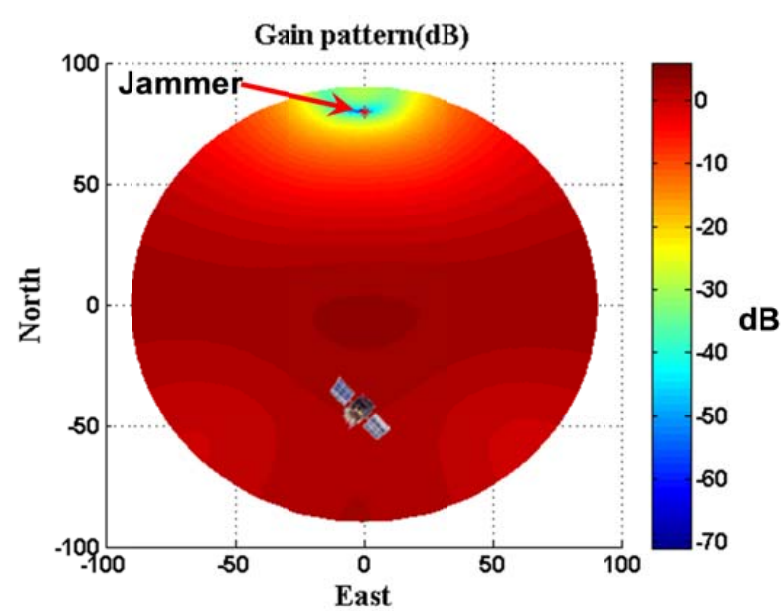

Fig. 8. Gain pattern with anti-jamming of power minimization. 


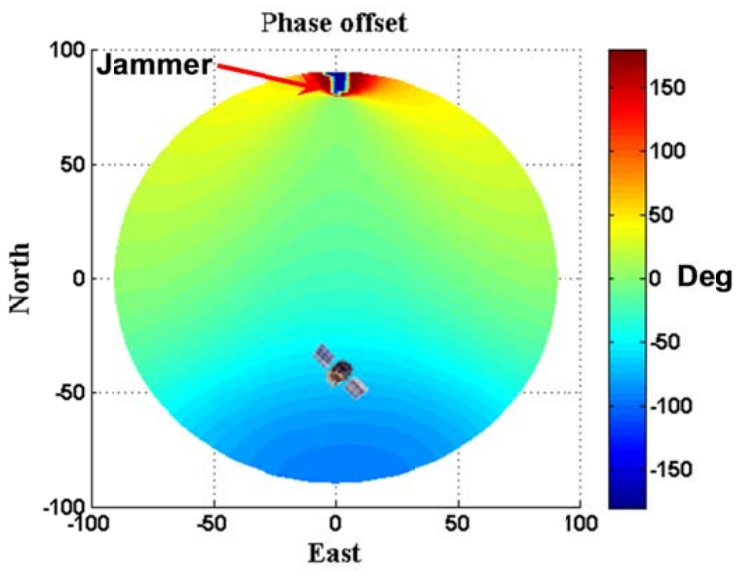

Fig. 9. Phase pattern with anti-jamming of power minimization.

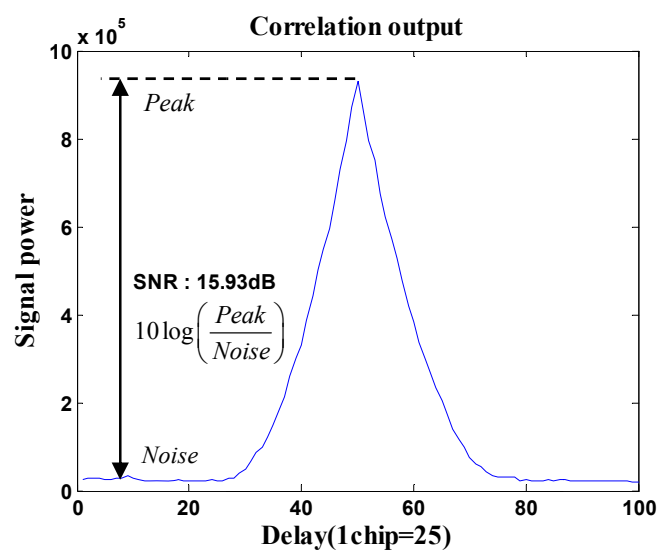

Fig. 10. SNR with anti-jamming of power minimization.

maintained at $0^{\circ}$ in the satellite direction. If beamforming is performed in a different satellite direction, the phase is maintained at $0^{\circ}$ in the direction, and a phase shift does not occur. The satellite signal SNR was calculated to be $21 \mathrm{~dB}$ as shown in Fig. 13. The value was $5 \mathrm{~dB}$ higher than that of the power minimization technique mentioned above, and was 6 $\mathrm{dB}$ higher than the input signal of a single antenna in Table 1. This was identical to the gain ( $6 \mathrm{~dB})$ of the four-element array antenna calculated using Eq. (12). Thus, it was also consistent with the theoretical value.

As shown above, the STAP beamforming technique, which forms a beam in the satellite direction, can prevent a phase shift as well as can enhance satellite signals. This technique conserves signals in the satellite direction, and enhances SNR. Therefore, it is an efficient technique which can improve anti-jamming performance.

On the other hand, the CCF was symmetric as shown in Figs. 10 and 13, which were the results of the STAP algorithm

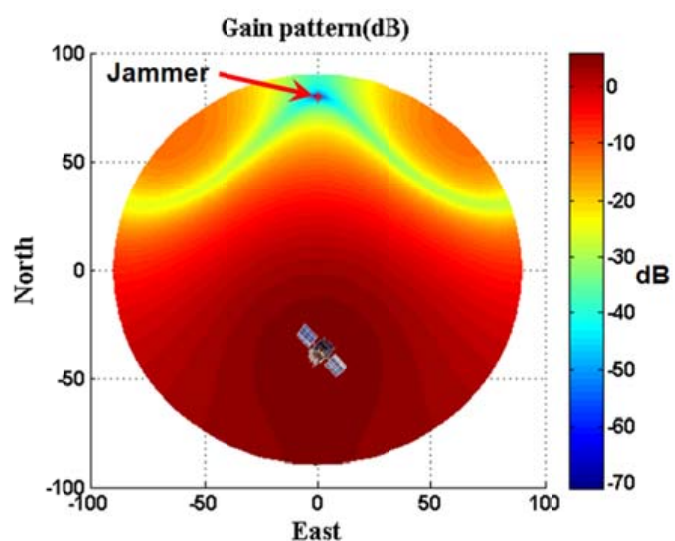

Fig. 11. Gain pattern with anti-jamming of beamforming.

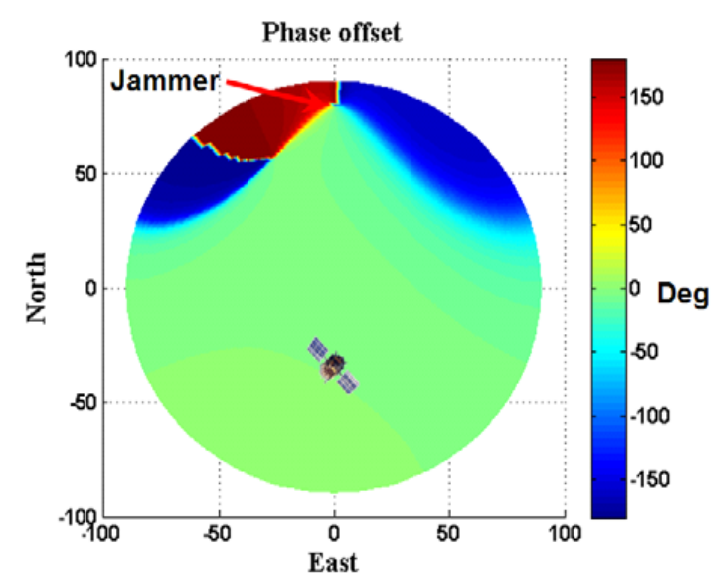

Fig. 12. Phase pattern with anti-jamming of beamforming.

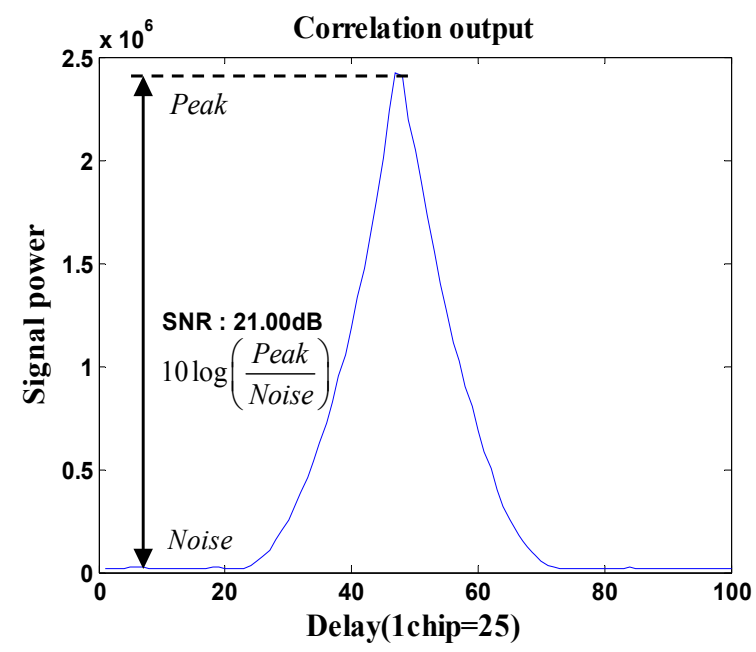

Fig. 13. SNR with anti-jamming of beamforming. 
that selected the center tap. Therefore, a pseudorange error did not occur similar to the results of Chapter 3.

\section{CONCLUSIONS}

In this study, an STAP beamforming algorithm for an array antenna system, which had been extended to time and space domains to increase the narrowband anti-jamming number, was analyzed. The cause of a pseudorange tracking error during the use of the STAP algorithm was theoretically analyzed, and a tap selection technique for preventing this was proposed. In addition, the performance analysis of the STAP algorithm, to which a beamforming algorithm had been added, was carried out in terms of SNR and phase measurements. It was found that the SNR was improved by the beamforming algorithm, and this could enhance antijamming performance. Also, the beamforming algorithm can prevent the shift of carrier phase measurements. Therefore, it could be applied to a reference position survey system or a precise navigation system.

\section{REFERENCES}

Applebaum, S. P. \& Chapman, D. J. 1976, Adaptive Arrays with Main Beam Constraints, IEEE Transactions on Antennas and Propagation, 24, 650-662

Bellanger, M. 2000, Digital Processing of Signals Theory and Practice, 3rd edition (Hoboken: Wiley)

Berefelt, F., Boberg, B., Eklöf, F., Malmström, J., Pääjärvi, L., et al. 2003, INS/GPS Integration with Adaptive Beamforming, ION GPS/GNSS 2003, 9-12 September, Portland, OR

Carlson, S. G., Popeck, C. A., Stockmaster, M. H., \& McDowell, C. E. 2003, Rockwell Collins' Flexible Digital Anti-Jam Architecture, ION GPS/GNSS 2003, 9-12 September, Portland, OR

Fante, R. L., Fitzgibbons, M. P., \& McDonald K. F. 2004, Effect of Adaptive Array Processing on GPS Signal Crosscorrelation, ION GNSS 17th International Technical Meeting of the Satellite Division, 21-24 September

Fante, R. L. \& Vaccaro, J. J. 2000, Wideband Cancellation of Interference in a GPS Receive Array, IEEE Transactions on Aerospace and Electronic systems, 36, 549-564

Frost, O. L. 1972, An Algorithm for Linearly Constrained Adaptive Array Processing, Proceedings of the IEEE, 60, 926-935. http://dx.doi.org/10.1109/PROC.1972.8817

Gupta, I. \& Ksienski, A. 1983, Effect of mutual coupling on the performance of adaptive arrays, IEEE Transactions on Antennas and Propagation, 31, 785-791. http:// dx.doi.org/10.1109/TAP.1983.1143128

Haefner, B., Naylor, J., \& Sorber, S. 2003, G-STAR, Lockheed Martin's Advanced GPS Anti-Jam Technology, ION 59th Annual Meeting, 23-25 June

Haykin, S. 1996, Adaptive Filter Theory, Third edition (New Jersey: Prentice Hall)

Johnson, D. H. \& Dudgeon, D. E. 1993, Array Signal Processing Concepts and Techniques (New Jersey: Prentice Hall)

Kaplan, E. D. \& Hegarty, C. J. 2006, Understanding GPS Principles and Applications. Second Edition (Boston: Artech House)

Lorenzo, D. S., Gautier, J., Rife, J., Enge, P., \& Akos, D. 2005, Adaptive Array Processing for GPS Interference Rejection, ION GNSS, September

Moore, T. D. 2002, Analytic study of space-time and spacefrequency adaptive processing for radio frequency interference suppression, PhD Dissertation, The Ohio State University

Parkinson, B. W., Spilker, J., \& Axelrad, P. 1996, The Global Positioning System: Theory and Applications (Washington: AIAA)

Rhee, J. H. \& Seo, J. 2013, eLoran Signal Strength and Atmospheric Noise Simulation over Korea, JKGS, 2, 101108. http://dx.doi.org/10.11003/JKGS.2013.2.2.101

Vo, A., Falchetti, C. R., \& Morrison, A. W. 2007, ADAP: Enhancing GPS Protection for NAVWAR, Proceedings of the 2007 National Technical Meeting of The Institute of Navigation, 22-24 January, 990-997

Ward, P. W. 1994, GPS Receiver RF Interference Monitoring, Mitigation, and Analysis Techniques, Journal of The Institute of Navigation, 41, 367-392.

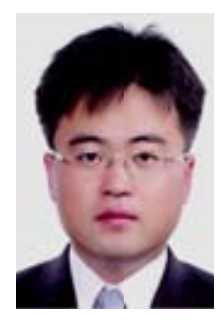

Kihoon Lee is a senior researcher at Agency for Defense Development. He received his B.S. from the Mechanical Engineering Department of POSTECH in 1999. He received his M.S. from the Mechanical Engineering Department of Korean Advanced Institute of Science and Technology in 2001. He has served as a researcher at Agency for Defense Development since 2001. His research focuses on the development of GNSS receiver, Anti-Jamming and Space Based Augmentation System. 


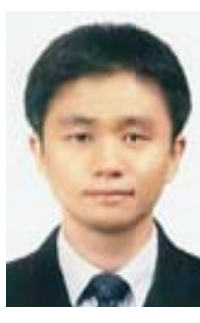

Hyoungmin So is a senior researcher of Agency for Defense Development (ADD) in Korea, Republic of. He received B.S. degree in mechanical engineering from Korea Univ. and M.S. and Ph.D. degree in aerospace engineering from Seoul National University (SNU). He worked in the field of GNSS and pseudolite receiver development including SDR and vector tracking loop algorithm in SNU GNSS laboratory. Since 2011, he's been working for ADD.

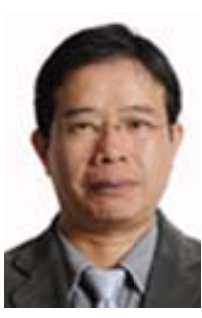

Kiwon Song is a principal researcher at Agency for Defense Development. He received his B.S. from the Electronical Engineering Department of Seonggyunkwan University in 1984. He received his M.S., Ph.D. degrees from the Electronical Engineering Department of Choongnam University in 1992 and 2002, respectively. He has served as a researcher at Agency for Defense Development since 1984. His research focuses on the satellite navigation system and GNSS signal processing. 